Original article

\title{
Characteristics of participants in the self-determined participation type care prevention program based on the differences in participation frequency: focusing on the Japan Science and Technology Agency Index of Competence
}

\author{
Suguru Ando ${ }^{1,2 *}$, Masaki Iwamura ${ }^{1}$, Yosuke Yamato $^{1}$, Kenji Shinbo $^{1}$, Wataru Nanikawa ${ }^{1,2}$, Hiroyuki Kajimoto ${ }^{1}$, \\ Hitoshi Kumada ${ }^{1}$ \\ ${ }^{1}$ Department of Physical Therapy, Faculty of Health Science, Aino University \\ ${ }^{2}$ Graduate School of Comprehensive Rehabilitation, Osaka Prefecture University
}

\section{ABSTRACT}

Objective: The purpose of this study was to investigate the characteristics of Submitted Mar. 11.2021 Accepted Jun. 17. 2021

participants based on the differences in participation frequency in the selfdetermined participation type care prevention program.

Methods: This study design was a cross-sectional study. Participants were 114 community-dwelling older adults aged 73.0 on median who were registered in the self-determined participation type care prevention program. Participants can freely decide the date of participation in this program. The exercise was performed depending on the ability of the participants. We evaluated program participation status, a higher-level functional capacity, grip strength, and walking speed. The higher-level functional capacity was evaluated using the Japan Science and Technology Agency Index of Competence (JST-IC). As an analysis, we first classified the frequency of participation into three groups: "less than once a week," "1-2 times a week," and "more than 3 times a week", and compared the outcomes. Secondly, we investigated the relationship between participation frequency and four categories of the JST-IC.

Results: Participants in the frequency of participation in "less than once a week," "1-2 times a week," and "more than 3 times a week" were 26, 76, and 12, respectively. The median scores of IST-IC were 13.0. There was no significant difference in IST-IC total, subscale scores and physical functions among the three groups based on the differences in participation frequency.

Conclusion: In the population with high higher-level functional capacity, there was no significant difference in higher-level functional capacity and physical function based on the frequency of participation in the self-determined participation type care prevention program. It was suggested that a longitudinal survey was necessary after unifying the implementation period of the participants. 


\section{INTRODUCTION}

Higher-level functional capacity is the ability of the communitydwelling older adults to live independently and actively. Lawton defined and systemized seven stages of competence for older adults, from the lowest and most basic function to the highest. The stages were, in ascending order of complexity, as follows: life maintenance, functional health, perception and cognition, physical self-maintenance, instrumental self-maintenance (instrumental activities of daily living; IADL), effectance, and social role ${ }^{1)}$. Among them, Lawton positioned IADL, effectance, and social role as higher-level functional capacity. The Tokyo Metropolitan Institute of Gerontology Index of Competence (TMIG-IC), which was developed based on Lawton's stage, was used to evaluate the higher-level functional capacity for older adults², 3). TMIG-IC is a quantified evaluation scale for the three high-level competences of the Lawton stage: IADL, intellectual activity (effectance in Lawton), and social role. Recently, several studies have used the Japan Science and Technology Agency Index of Competence (JSTIC) to assess higher-level functional capacity in Japan ${ }^{4 \cdot 6}$. The JSTIC is an index developed by Suzuki et al. that measures the ability of older adults to live their daily lives independently and actively in the community ${ }^{7-9)}$. The evaluation scale of JST-IC considers the lifestyles of modern older adults (e.g., the use of mobile phones) that cannot be evaluated by TMIG-IC. The evaluation of higherlevel functional capacity using the JST-IC is essential for the early prevention of care and reducing social isolation among current older adults due to the changes in their living environment and lifestyle.

Many care prevention programs have been implemented to prevent long-term care and social isolation in Japan. Participation in the care prevention program is not only expected to improve physical function but also reduce social isolation and the lack of social interaction. Implementation of various care prevention programs reduces the need for new care-need certification ${ }^{10-12)}$ and improves the oral and physical function and the quality of life of older adults ${ }^{13,14}$. In the comprehensive support project introduced in 2015, the distinction between primary and secondary prevention has been abolished, and effective care prevention efforts are being promoted according to the actual conditions of the community. According to the survey results in $2018,92.6 \%$ of the municipalities conducted long-term care prevention classes, and although the environment for promoting long-term care prevention for community-dwelling older adults has begun to be established nationwide ${ }^{15}$. However, most classrooms in the care prevention program had an exercise schedule planned at a predetermined frequency. A systematic review of improving exercise compliance to prevent falls reported that incorporating exercise into daily activities to promote habitual involvement is important ${ }^{16}$. Therefore, it is necessary to have a care prevention program that allows self-determined participation to some extent, which reflects the independence of the community-dwelling older adults.

Ibaraki City, Osaka Prefecture, maintains a self-determined participation-type care prevention program. Participants in this program can self-determine how often and when they will participate in the program. A characteristic of this care prevention program is that participants can join the program twice a weekday in the morning and afternoon at any time if they register. Participation frequency depends on individual schedules and interactions; therefore, participation and continuation of the program should be self-determined, reflecting autonomy. Previous studies reported that exercises incorporated into daily lifestyle had high adherence and was suggested as a promising alternative to formal training ${ }^{17,18}$. We believe that self-determined participation programs that promote independent decision-making will be useful for reducing social isolation and the reluctance of social participation. However, the participants' characteristics based on the frequency of participation in this self-determined participation program have not been examined; for example, more participation in the program affects physical functions and higher-level functional capacity. By investigating the characteristics of participation in this self-determined participation type care prevention program based on the frequency of participation, it is possible to know how the frequency of community-dwelling older adults spending in the long-term care prevention program is related to physical function and higher-level functional capacity. In addition, it may be possible to verify the effect of this program from 
the viewpoint of participation frequency through future longitudinal surveys and comparison with general long-term care prevention programs.

Thus, we hypothesized that registrants who participate frequently according to their convenience have more opportunities to exercise and may have higher physical function than registrants who participate less. Additionally, they may have the higher abilities needed to become independent and lead an active daily life. This study investigated the characteristics of participants based on the differences in participation frequency in the self-determined participation type care prevention program held throughout the year.

\section{Materials and Methods}

Study Design and Participants

This was a cross-sectional study of community-dwelling older adults in Ibaraki city, Osaka prefecture, Japan. Participants were community-dwelling older adults enrolled in the care prevention program held at the Ibaraki city healthcare center. All registrants were invited to participate by mail. All 184 registrants agreed to participate in the survey, which was conducted in two phases. The first survey was conducted from August to September 2017, and the second survey was conducted from February to March 2018. All measurements were conducted at the Ibaraki city healthcare center.

The inclusion criteria for our study were as follows: aged 65 years and the ability to understand and follow our instructions. The exclusion criteria were as follows: those with an implanted pacemaker, systolic blood pressure $\geq 180 \mathrm{mmHg}$, or diastolic blood pressure $\geq 120 \mathrm{mmHg}$ immediately before the survey, and those who have already participated in the first survey at the time of the second survey. The research ethics committee of Aino University (ref no. 2017-006) approved this study. All participants provided written informed consent.

\section{Ibaraki City Care Prevention Program}

The Ibaraki City Care Prevention Program is held on weekdays throughout the year at the Ibaraki city healthcare center. Registration for participation is announced through the city's public relations magazine. Applicants can enroll in the program if they are aged 65 years or above, living in Ibaraki city, and meet the inclusion criteria. Registrants can freely decide the date of participation in this program and can attend the 90 -minute program twice a day at any time. The registration criteria for not being able to enroll in this care prevention program were: (1) myocardial infarction, stroke (within 6 months), (2) angina/heart failure/severe arrhythmia, (3) hypertension (systolic blood pressure $\geq 180 \mathrm{mmHg}$ or diastolic blood pressure $\geq 120 \mathrm{mmHg}$ ), (4) shortness of breath due to chronic obstructive pulmonary disease, (5) serious complications due to diabetes mellitus, (6) acute arthritis, and/or (7) acute pneumonia/hepatitis, besides (8) a risk of physical condition due to exercise. The program included resistance training and aerobic dance. The exercise class included warm-up and cooling-down for 20 minutes before and after exercise. Aerobic dance consisted of whole-body exercises that incorporated the movements of various joints involving the arms and legs, and the trunk. Resistance exercises consisted of painless progressive exercises using their body weight. All exercises were performed at a "very lightsomewhat hard" intensity, depending on the participants' abilities (between 9 and 13 on the Borg scale). Exercise instructions were given by an experienced staff member.

Procedure

All participants had their height and body weight measured. Body mass index (BMI) was calculated using height and body weight, and we also measured blood pressure. The blood pressure was measured in a right arm using an automatic blood pressure measurement system. Using questionnaires, we examined the participants' basic characteristics, including age, sex, marital status, drinking habits, smoking habits, hospitalization history, current medical history, participation frequency, and participation period. Subsequently, the higher-level functional capacity was measured using another questionnaire. Regarding the frequency and duration of participation, we asked about their situation at the time of the first or second survey. For the participation frequency, we prepared the following three answers: less than once a week, 1-2 times a week, and more than 3 times a week. Participants responded to their 
frequency of participation in this care program at the time of our survey. We divided the participants into three groups and used them in the analysis according to these three answers. For the duration of participation, we asked for the duration from the date of first participation in this program. We have prepared the following four answers: Less than a month, 1-3 months, 3-6 months, and 6 months or more. At the time of the first and second surveys, participants had different periods of participation due to the different start times of participation in this care prevention program. In addition, participants responded to the periods of participation at the time we measured their basic characteristics and physical function. Finally, all participants were assessed for grip strength, walking speed, and appendicular skeletal muscle mass as physical functions.

\section{Assessments}

The higher-level functional capacity was evaluated using a questionnaire-based scale called JST-IC ${ }^{19,20)}$. The JST-IC contains four categories regarding 'usage of technology', 'collecting information and health literacy', 'daily life management' and 'social participation'7, 9). Furthermore, a questionnaire was constructed with 16 items under four factors (four items per category) identified through factor analysis. The score of the JSTIC was the number of "Yes" answers, with a maximum score of 16 points; higher scores indicated higher competence. Grip strength was measured twice in a standing position using a dynamometer (Digital Handgrip Meter KEEP, MACROSS Inc. Tokyo, Japan). The maximum value was adopted as the representative value. Walking speed was measured in seconds using a stopwatch by one researcher. Participants walked once at a comfortable walking speed for $6 \mathrm{~m}$. The walkway was a flat surface with two markers kept at the start and end of the 6-m path, and the acceleration and deceleration paths were set $1 \mathrm{~m}$ apart from the $6 \mathrm{~m}$ path. Appendicular skeletal muscle mass was assessed using multifrequency bioelectrical impedance analysis using the InBody S10 (InBody Japan, Tokyo, Japan). The InBody S10 uses a tetrapolar, eight-point tactile electrode system that separately measures impedance of the arms, trunk, and legs at 6 different frequencies $(1,5,50,250,500$, and 1,000 $\mathrm{kHz})$ for each segment.
The posture during measurement involved standing barefoot on the measuring equipment. The pinching-type electrodes were attached to the thumb and middle fingers of the upper extremities and to the heels of the lower extremities. Skeletal muscle mass was derived as the sum of the muscle mass of the four limbs, and the skeletal muscle index (SMI; $\mathrm{kg} / \mathrm{m}^{2}$ ) was calculated.

\section{Statistical Analysis}

Descriptive statistics were calculated for all variables. Normality was evaluated using the Shapiro-Wilk test. Data are presented as mean \pm standard deviation or median (interquartile range [IQR]) or frequency (percentage). Participation frequency was divided into three groups: "less than once a week," "1-2 times a week," and "more than 3 times a week". To compare the values of the three groups, a Kruskal-Wallis test for continuous measures and Fisher-Freeman-Halton exact tests for proportions were performed. Additionally, as a secondary analysis, the KruskalWallis test was used to compare the total scores for each of the four categories among the three groups. We also calculated the effect size. Effect size $r$ and Cramer's $V$ were calculated for the results of the Kruskal-Wallis test and Fisher-Freeman-Halton exact tests, respectively. We considered small, medium, and large effect sizes to be indicated by $r$ or $V$ values of $0.1,0.3$, and 0.5 , respectively. Regarding effect size $r$, post hoc tests were conducted using Bonferroni-adjusted Mann-Whitney U tests for multiple pairwise comparisons. The effect size $r$ between groups was calculated as $r$ $=\mathrm{Z} / \sqrt{ } \mathrm{n}$. Cramer's $V$ was output with Fisher-Freeman-Halton exact tests results using the statistical software. All statistical analyses were performed using SPSS version 27 for Windows (IBM Japan Corporation, Tokyo, Japan). The level of statistical significance was set at $p<0.05$.

\section{RESULTS}

Figure 1 shows the flowchart for selecting the participants in this study. About 91 and 93 registrants participated in the first and second surveys, respectively. Participants in the first survey were excluded from further analyses due to the following reasons: 


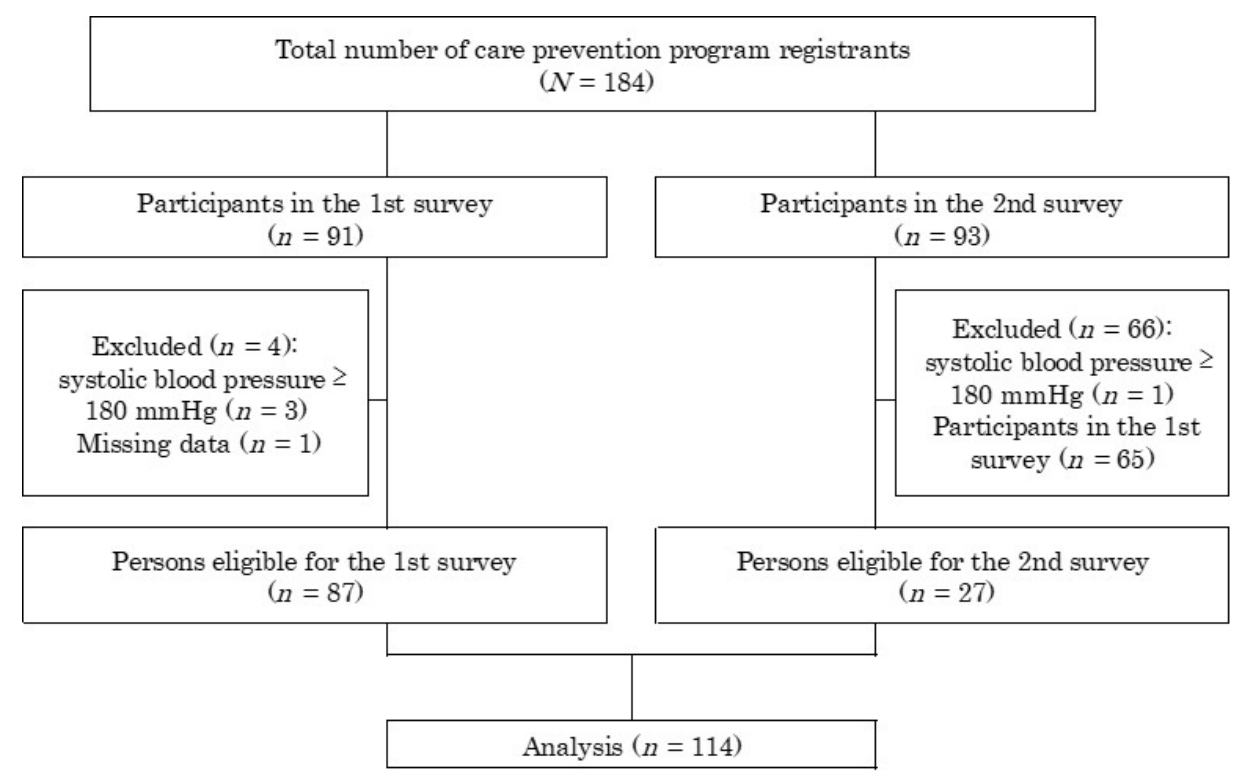

Figure 1. Participant selection flowchart

(1) hypertension (systolic blood pressure $\geq 180 \mathrm{mmHg}$ ) $(\mathrm{n}=3$ ), and

(2) missing data $(\mathrm{n}=1)$. Participants in the second survey were excluded due to the following reasons: (1) hypertension (systolic blood pressure $\geq 180 \mathrm{mmHg})(\mathrm{n}=1)$, and (2) participated in the first survey $(n=65)$. The final analysis included 114 registrants who participated in the first and second surveys.

Table 1 shows the participants' basic characteristics, the overall outcome measures, participation frequency, and the results of the three-group comparison. The median age of the study participants was 73.0 years (IQR, 69.8-76.0 years), and 71.9\% were women. Participants who are continuing this care prevention program for more than 6 months were $86(75.4 \%)$. The median scores of JSTIC were 13.0. There were no significant differences in basic characteristics and physical function or JST-IC between the three groups.

Table 2 shows the results of the secondary analysis. According to the result of the Kruskal-Wallis test, there was no significance in the participation frequency in all categories of JST-IC.

\section{DISCUSSION}

This study found that there was no significant difference in physical function or higher-level functional capacity based on the participation frequency in a self-determined participation type care prevention program. This is the first report to show the characteristics of participants by participation frequency in a selfdetermined participation type care prevention program.

An essential characteristic of self-determined participation type care prevention programs is that the registrants do not have to attend on a predetermined mandatory day. The registrants decide to participate in the program themselves. In other words, the selfdetermined participation type care prevention program, compared to other prevention programs, does not impose compulsory participation in the program and is based on the registrants' free will. A motor function improvement program for long-term care prevention in Japan recommends that it should be conducted at least twice a week for three months ${ }^{21)}$. A review of strength training for older adults also reported improvements in lower limb strength and walking speed with high-intensity training, two or three times per week $^{22)}$. However, in our study, there was no significant difference based on the participation frequency between groups in the physical function. The effect of physical function was not investigated in our cross-sectional study, but it was speculated that the cause was low exercise frequency and exercise intensity. Concerning the group of "less than once a week" there is a possibility that has been affected by lack of exercise frequency. Participation at least twice a week 


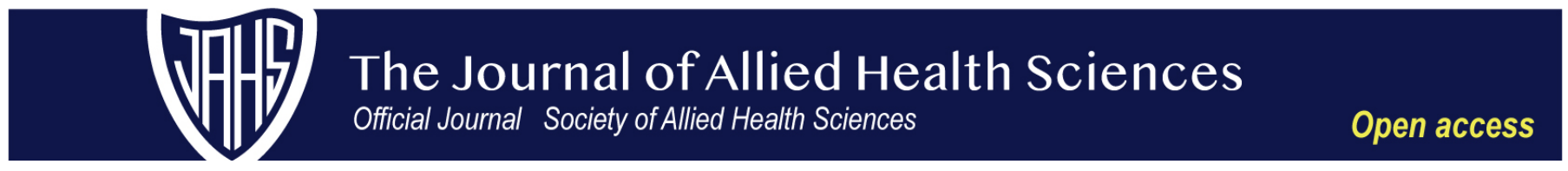

\begin{tabular}{|c|c|c|c|c|c|c|}
\hline Variable & $\begin{array}{c}\text { Overall } \\
(\mathrm{n}=114)\end{array}$ & $\begin{array}{c}<1 \text { time/week } \\
(\mathrm{n}=26)\end{array}$ & $\begin{array}{c}1-2 \text { times/week } \\
(\mathrm{n}=76)\end{array}$ & $\begin{array}{c}\geq 3 \text { times/week } \\
(\mathrm{n}=12)\end{array}$ & $p$ value* & Effect size** \\
\hline \multirow[t]{3}{*}{ Age (years), median } & $73.0(69.8-76.0)$ & $72.0(68.8-76.0)$ & $73.0(70.0-76.0)$ & $72.0(69.3-74.8)$ & 0.906 & $0.037^{\mathrm{a}}$ \\
\hline & & & & & & $0.032^{\mathrm{b}}$ \\
\hline & & & & & & $0.003^{\mathrm{c}}$ \\
\hline Women, $n(\%)$ & $82(71.9)$ & $19(73.1)$ & $54(71.1)$ & $9(75.0)$ & 1.000 & 0.030 \\
\hline \multirow[t]{3}{*}{ Height $(\mathrm{cm})$} & $156.3 \pm 6.9$ & $157.4 \pm 7.0$ & $155.9 \pm 7.1$ & $156.3 \pm 6.2$ & 0.794 & $0.068^{\mathrm{a}}$ \\
\hline & & & & & & $0.008^{\mathrm{b}}$ \\
\hline & & & & & & $0.054^{\mathrm{c}}$ \\
\hline \multirow[t]{3}{*}{ Weight $(\mathrm{kg})$} & $55.2 \pm 9.2$ & $53.6 \pm 9.2$ & $55.2 \pm 9.2$ & $58.2 \pm 9.0$ & 0.287 & $0.076^{\mathrm{a}}$ \\
\hline & & & & & & $0.135^{\mathrm{b}}$ \\
\hline & & & & & & $0.237^{\mathrm{c}}$ \\
\hline \multirow[t]{3}{*}{$\operatorname{BMI}\left(\mathrm{kg} / \mathrm{m}^{2}\right)$} & $22.5 \pm 3.0$ & $21.5 \pm 2.6$ & $22.6 \pm 3.0$ & $23.8 \pm 3.4$ & 0.115 & $0.147^{\mathrm{a}}$ \\
\hline & & & & & & $0.119^{\mathrm{b}}$ \\
\hline & & & & & & $0.326^{\mathrm{c}}$ \\
\hline Marital status, $n(\%)$ & $79(69.3)$ & $21(80.8)$ & $52(68.4)$ & $6(50.0)$ & 0.167 & 0.181 \\
\hline Drinking habits, $n(\%)$ & $22(19.3)$ & $6(23.1)$ & $13(17.1)$ & $3(25.0)$ & 0.620 & 0.080 \\
\hline Smoking habits, $n(\%)$ & $5(4.4)$ & $0(0.0)$ & $5(6.6)$ & $0(0.0)$ & 0.468 & 0.151 \\
\hline Hospitalization history (past 1 year), $n$ (\%) & $12(10.5)$ & $3(11.5)$ & $8(10.5)$ & $1(8.3)$ & 1.000 & 0.028 \\
\hline \multicolumn{7}{|l|}{ Current medical history, $n(\%)$} \\
\hline Nervous system disorders & $3(2.6)$ & $2(7.7)$ & $1(1.3)$ & $0(0.0)$ & 0.257 & 0.174 \\
\hline Musculoskeletal disorders & $14(12.3)$ & $2(7.7)$ & $11(14.5)$ & $1(8.3)$ & 0.745 & 0.095 \\
\hline Cardiac disorders & $32(28.1)$ & $5(19.2)$ & $20(26.3)$ & $7(58.3)$ & 0.054 & 0.240 \\
\hline Endocrine and metabolic disorders & $27(23.7)$ & $5(19.2)$ & $20(26.3)$ & $2(16.7)$ & 0.741 & 0.089 \\
\hline Digestive disorders & $8(7.0)$ & $3(11.5)$ & $5(6.6)$ & $0(0.0)$ & 0.513 & 0.124 \\
\hline Neoplasm & $1(0.9)$ & $1(3.8)$ & $0(0.0)$ & $0(0.0)$ & 0.333 & 0.173 \\
\hline Respiratory disorders & $3(2.6)$ & $2(7.7)$ & $1(1.3)$ & $0(0.0)$ & 0.257 & 0.174 \\
\hline Infections & $1(0.9)$ & $0(0.0)$ & $1(1.3)$ & $0(0.0)$ & 1.000 & 0.067 \\
\hline Reproductive system disorders & $3(2.6)$ & $2(7.7)$ & $1(1.3)$ & $0(0.0)$ & 0.257 & 0.174 \\
\hline Eye disorders & $4(3.5)$ & $1(3.8)$ & $3(3.9)$ & $0(0.0)$ & 1.000 & 0.065 \\
\hline \multicolumn{7}{|l|}{ Participation period, $n(\%)$} \\
\hline Less than a month & $8(7.0)$ & $1(3.8)$ & $7(9.2)$ & $0(0.0)$ & 0.864 & 0.116 \\
\hline $1-3$ months & $13(11.4)$ & $2(7.7)$ & $9(11.8)$ & $2(16.7)$ & & \\
\hline 3-6 months & $7(6.1)$ & $1(3.8)$ & $5(6.6)$ & $1(8.3)$ & & \\
\hline 6 months or more & $86(75.4)$ & $22(84.6)$ & $55(72.4)$ & $9(75.0)$ & & \\
\hline \multirow[t]{3}{*}{ JST-IC (scores), median } & $13(11.0-14.3)$ & $14(11.0-15.0)$ & $13(12.0-14.0)$ & $11(9.0-14.0)$ & 0.092 & $0.071^{\mathrm{a}}$ \\
\hline & & & & & & $0.204^{\mathrm{b}}$ \\
\hline & & & & & & $0.336^{\mathrm{c}}$ \\
\hline \multirow[t]{3}{*}{ Grip strength $(\mathrm{kg})$, median } & $24.6(21.7-30.7)$ & $24(22.8-28.5)$ & $26(21.0-33.3)$ & $25(22.0-34.0)$ & 0.898 & $0.048^{\mathrm{a}}$ \\
\hline & & & & & & $0.116^{\mathrm{b}}$ \\
\hline & & & & & & $0.194^{\mathrm{c}}$ \\
\hline \multirow[t]{3}{*}{ Walking speed (m/seconds) } & $1.4 \pm 0.2$ & $1.4 \pm 0.2$ & $1.4 \pm 0.2$ & $1.3 \pm 0.3$ & 0.448 & $0.018^{\mathrm{a}}$ \\
\hline & & & & & & $0.027^{\mathrm{b}}$ \\
\hline & & & & & & $0.112^{\mathrm{c}}$ \\
\hline \multirow[t]{3}{*}{ SMI $\left(\mathrm{kg} / \mathrm{m}^{2}\right)$, median } & $6.2(5.6-6.8)$ & $6(6.0-7.0)$ & $6(6.0-7.0)$ & $7(6.0-7.0)$ & 0.753 & $0.056^{\mathrm{a}}$ \\
\hline & & & & & & $0.041^{b}$ \\
\hline & & & & & & $0.112^{\mathrm{c}}$ \\
\hline
\end{tabular}

Data are presented in mean \pm standard deviation or median (interquartile range) or frequency (percentage). *Kruskal-Wallis test for continuous measures and Fisher-Freeman-Halton exact tests for proportions. JST-IC: Japan Science and Technology Agency Index of Competence; SIM: skeletal muscle index. **Effect size between groups was calculated as $r$ for Kruskal-Wallis test and Cramer's V for Fisher-Freeman-Halton exact tests. a: Effect size $r$ derived from post hoc test by Bonferroni-adjusted Mann-Whitney U test for multiple pairwise comparison of " $<1$ time/week" versus "1-2 times/week." b: Effect size $r$ derived from post hoc test by Bonferroni-adjusted Mann-Whitney U test for multiple pairwise comparison of "1-2 times/week" versus " $\geq 3$ times/week." c: Effect size $r$ derived from post hoc test by Bonferroni-adjusted Mann-Whitney $U$ test for multiple pairwise comparison of " $<1$ time/week" versus " $\geq 3$ times/week." 


\begin{tabular}{|c|c|c|c|c|c|}
\hline Variable & $\begin{array}{c}<1 \text { time } / \text { week } \\
(\mathrm{n}=26)\end{array}$ & $\begin{array}{c}1-2 \text { times } / \text { week } \\
(\mathrm{n}=76)\end{array}$ & $\begin{array}{c}\geq 3 \text { time/week } \\
(\mathrm{n}=12)\end{array}$ & $p$ value & Effect size $r^{*}$ \\
\hline \multirow[t]{3}{*}{ Usage of technology } & $4.0(4.0-4.0)$ & $4.0(3.0-4.0)$ & $3.5(3.0-4.0)$ & 0.150 & $0.101^{*}$ \\
\hline & & & & & $0.139^{b}$ \\
\hline & & & & & $0.194^{e}$ \\
\hline \multirow[t]{3}{*}{ Collecting information and health literacy } & $4.0(2.8-4.0)$ & $4.0(3.0-4.0)$ & $3.0(3.0-4.0)$ & 0.455 & $0.015^{2}$ \\
\hline & & & & & $0.126^{b}$ \\
\hline & & & & & $0.091^{\mathrm{c}}$ \\
\hline \multirow[t]{3}{*}{ Daily life management } & $4.0(3.0-4.0)$ & $4.0(3.0-4.0)$ & $3.0(2.0-3.8)$ & 0.087 & $0.060^{2}$ \\
\hline & & & & & $0.186^{b}$ \\
\hline & & & & & $0.209^{2}$ \\
\hline \multirow[t]{3}{*}{ Social participation } & $3.0(1.0-4.0)$ & $2.0(1.0-3.0)$ & $1.0(1.0-3.0)$ & 0.141 & $0.074^{2}$ \\
\hline & & & & & $0.173^{b}$ \\
\hline & & & & & $0.176^{e}$ \\
\hline
\end{tabular}

Data are presented in median (interquartile range). JST-IC: Japan Science and Technology Agency Index of Competence. *Effect size between groups was calculated as $\mathrm{r}$ for Kruskal-Wallis test. a: Effect size $\mathrm{r}$ derived from post hoc test by Bonferroni-adjusted Mann-Whitney U test for multiple pairwise comparison of " $<1$ time/week" versus "1-2 times/week." b: Effect size $r$ derived from post hoc test by Bonferroni-adjusted Mann-Whitney U test for multiple pairwise comparison of "1-2 times/week" versus " $\geq 3$ times/week." c: Effect size $r$ derived from post hoc test by Bonferroni-adjusted Mann-Whitney U test for multiple pairwise comparison of " $<1$ time/week" versus " $\geq 3$ times/week."

may be required, as in previous studies ${ }^{21)}$. There was no significant difference in physical function in groups that participated more than once a week as compared with the "less than once a week" group. Exercise intensity may have been the reason for these. In previous studies, it was recommended to gradually increase exercise intensity and exercise load every month ${ }^{21}$. In this care program, exercise intensity was determined according to individual physical fitness levels. However, their exercise load may have been inadequate for improving physical function. Furthermore, in a longitudinal study investigating the relationship between physical function and higher-level functional capacity, it was reported that a decrease in walking speed was a risk of decline in higher-level functional capacity ${ }^{23}$. In the future, it is necessary to verify whether the physical function improved by appropriate exercise load can improve the higher-level functional capacity.

There were no significant differences between groups in higherlevel functional capacity in present study. In our result, JST-IC had a median of 13.0. In contrast, the average score of JST-IC, which surveyed 1,306 community-dwelling older adults, was 9.57). Our results (median age 73.0) are higher than the average values from participants aged 74.0 years reported from a previous study of older adults in the general population. The participants of our study were presumed to be community-dwelling older adults with high higherlevel functional capacity. There was no difference in the diseases, physical functions, and periods of participation in this care prevention program that restricted the activity, so we inferred that there was no significant difference in JST-IC in the frequency of participation in the care prevention program. A previous study that described choices reported that people are more effective in learning when they can make their own choices than when they are forced to make choices ${ }^{24)}$. Regarding self-management, selfdetermined choices may influence one's actions positively. However, participants with higher-level functional capacity in this study may have had lower exercise loads undertaken in a selfdetermined participation type care prevention program. Therefore, it did not contribute to the improvement of physical function and was not related to higher-level functional capacity.

The four categories of JST-IC contain the subscales necessary for older adults to live independently. It is important to investigate the higher-level functional capacity of community-dwelling older adults not only in the total score of JST-IC but also in the detailed category consisting of IADL, effectance, and social role. However, there was no significant difference in the frequency of participation in the four categories of JST-IC among the participants in this study. On the other hand, in previous studies, the gender-specific subscales (men, women) of JST-IC for community-dwelling older adults aged 65-74 were 2.8 and 2.4 for usage of technology, 3.1 and 2.9 for collecting information and health literacy, 3.1 and 3.1 for daily life management, and 1.9 and 1.8 for social participation, respectively ${ }^{7}$. The results of our secondary analysis of JST-IC 
subscales were 3.0-4.0 for usage of technology, 3.0-4.0 for collecting information and health literacy, 3.0-4.0 for daily life management, and 1.0-3.0 for social participation, respectively. Although no statistical comparisons were performed, it might indicate that participants in this study were active in each category as well as the JST total score in the results of our secondary analysis.

This study had certain limitations. We did not compare the selfdetermined participation type care prevention program to a common care prevention program with a predetermined participation frequency. Therefore, we have not investigated the effect of a self-determined participation type care prevention program on the JST-IC. Next, we were unable to unify the period from the first day of participation in this program to measurement date for our survey, because we conducted our survey during the care prevention program that was already held. In the future, we should unify the participation period in this program and investigate. In addition, we did not hear personal factors, purpose or motivation related to the frequency of participation in the program. We also did not consider environmental factors, such as the distance from the participant's home and the transportation to the Ibaraki city healthcare center and the seasonality of the first and second surveys. A previous study has reported that the shorter the distance to the place where the long-term care prevention project was implemented, the more frequently the facility was used ${ }^{25)}$. In another previous study, it was reported that physical activity depends on the season ${ }^{26}$. The influence of personal or environmental factors may have changed the frequency of their participation in the program.

\section{CONCLUSION}

Participants in our study had high higher-level functional capacity. Our findings showed that in the population with high higher-level functional capacity, there was no significant difference in higher-level functional capacity and physical function based on the frequency of participation in the self-determined participation type care prevention program. In the future, we should unify the conditions such as unifying the implementation period of the program and investigate the characteristics of participants due to the difference in participation frequency in a longitudinal survey.

\section{CONFLICT OF INTEREST}

The authors have no conflicts of interest to declare.

\section{ACKNOWLEDGEMENTS}

The authors would like to offer our special thanks to the participants who cooperated in our study and the Ibaraki City Office for assistance with participant recruitment.

\section{REFERENCES}

1. Lawton MP: Assessing the competence of older people. In: Kent DP, Kastenbaum R, Sherwood S (eds): Research, planning, and action for elderly: the power and potential of social science, pp. 122-143, Human Science Press, New York, 1972

2. Koyano W, Shibata H, Nakazato K, et al.: Measurement of competence in the elderly living at home: Development of an index of competence. Nippon Koshu Eisei Zasshi 34: 109-114, 1987 [in Japanese]

3. Koyano W, Shibata H, Nakazato K, et al:: Measurement of competence: Reliability and validity of the TMIG Index of Competence. Arch Gerontol Geriatr 13: 103-116, 1991

4. Maruya $\mathrm{K}$, Fujita $\mathrm{H}$, Arai T, et al.: Identifying elderly people at risk for cognitive decline by using the 2-step test. J Phys Ther Sci 30(1): 145-149, 2018

5. Takahashi J, Kawai H, Suzuki $\mathrm{H}$, et al.: Reliability and validity of the activity diversity questionnaire for older adults in Japan. Int $J$ Environ Res Public Health 17(7): 1-9, 2020

6. Takatori K, Matsumoto D, Miyazaki M, et al.: The difference between self-perceived and chronological age in the elderly may correlate with general health, personality and the practice of good health behavior: A cross-sectional study. Arch Gerontol Geriatr 83: 13-19, 2019

7. Iwasa $\mathrm{H}$, Masui $\mathrm{Y}$, Inagaki $\mathrm{H}$, et al.: Assessing competence at a higher level among older adults.: Development of the Japan Science and Technology Agency Index of Competence (JST-IC). Aging Clin Exp Res 30: 383-393, 2018 
8. Iwasa $\mathrm{H}$, Masui $\mathrm{Y}$, Inagaki $\mathrm{H}$, et al.: Development of the Japan Science and Technology Agency Index of Competence (JST-IC) to assess functional capacity in older adults: Conceptual definitions and preliminary items. Gerontol Geriatr Med 1:

2333721415609490, 2015

9. Suzuki T: Health status of older adults living in the community in Japan: Recent changes and significance in the super-aged society. Geriatr Gerontol Int 18(5): 667-677, 2018

10. Yamada M, Arai H: Self-Management Group Exercise Extends Healthy Life Expectancy in Frail Community-Dwelling Older Adults. Int. J. Environ. Res. Public Health 14: 531. doi:10.3390/ijerph14050531., 2017

11. Kato C, Fujita R, Ida K, et al.: Participant characteristics and intervention effects of a program to improve the physical function in community-dwelling frail elderly subjects. Nippon Ronen Igakkai Zasshi 50: 804-811, 2013 [in Japanese]

12. Fujimoto $S$, Yamazaki $S$, Wakabayashi A, et al.: The effects of Taichi exercise for the prevention of long-term care in communitydwelling frail elderly people-New care-need certification and mortality-. Nippon Ronen Igakkai Zasshi 48: 699-706, 2011 [in Japanese]

13. Iwao Y, Shigeishi H, Takahashi S, et al.: Improvement of physical and oral function in community-dwelling older people after a 3month long-term care prevention program including physical exercise, oral health instruction, and nutritional guidance. Clin Exp Dent Res 5(6): 611-619, 2019

14. Yuri Y, Takabatake $S$, Nishikawa T, et al.: The effects of a life goalsetting technique in a preventive care program for frail communitydwelling older people: A cluster nonrandomized controlled trial. BMC Geriatr 16(1): 1-11, 2016

15. Ministry of Health, Labor and Welfare, Japan: Survey results on the implementation status of the care prevention project and the care prevention / daily life support comprehensive project (community support project) 2018.

http://www.mhlw.go.jp/content12300000/000570876.pdf (accessed Dec 20, 2020)

16. Hughes KJ, Salmon N, Galvin R, et al.: Interventions to improve adherence to exercise therapy for falls prevention in communitydwelling older adults: systematic review and meta-analysis. Age Aging 48: 185-195, 2019

17. Clemson L, Fiatarone Singh MABundy A, et al.: Integration of balance and strength training into daily life activity to reduce rate of falls in older people (the LiFE study): randomised parallel trial. BMJ 345: e4547, 2012
18. Weber M, Belala N, Clemson L, et al.: Feasibility and Effectiveness of Intervention Programmes Integrating Functional Exercise into Daily Life of Older Adults: A Systematic Review. Gerontology 64(2):172-187, 2018

19. Fukutomi E, Okumiya K, Wada T, et al.: Importance of cognitive assessment as part of the "KihonChecklist" developed by the Japanese Ministry of Health, Labor and Welfare for prediction of frailty at a 2-year follow up. Geriatr Gerontol Int 13(3): 654-662, 2013

20. Tomata $\mathrm{Y}$, Sugiyama K, Kaiho $\mathrm{Y}$, et al.: Predictive ability of a simple subjective memory complaints scale for incident dementia: Evaluation of Japan's national checklist, the "Kihon Checklist". Geriatr Gerontol Int 17(9): 1300-1305, 2017

21. Ministry of Health, Labor and Welfare, Japan: Long-term care prevention manual. Revised edition. https://www.mhlw.go.jp/topics/2009/05/d//tp0501-1_1.pdf (accessed Sep 14, 2020)

22. Liu CJ, Latham NK: Progressive resistance strength training for improving physical function in older adults. Cochrane Database Sys Rev 3: doi: 10.1002/14651858.CD002759.pub2, 2009

23. Nakamoto M, Otsuka R, Yuki A, et al.: Higher gait speed and smaller sway area decrease the risk for decline in higher-level functional capacity among middle-aged and elderly women. Arch Gerontol Geriatr 61: 429-436, 2015

24. Chambon $\mathrm{V}$, Théro $\mathrm{H}$, Vidal $\mathrm{M}$, et al.: Information about action outcomes differentially affects leaming from self-determined versus imposed choices. Nat Hum Behav 4: 1067-1079, 2020

25. Hirai $\mathrm{H}$, Kondo $\mathrm{K}$ : Related factors in the elderly's use of municipal institutions: basic study for promoting participation in a care prevention program. Nihon Koshu Eisei Zasshi 55(1): 37-45, 2008 [in Japanese]

26. Ysunaga A, Togo F, Watanabe E, et al.: Sex, age, season, and habitual physical activity of older Japanese: the Nakanojo study. Aging Phys Act 16(1): 3-13, 2008 